\title{
Efeito de um programa de aprimoramento das habilidades de comunicação oral na ansiedade e no estresse autorreferidos
}

\author{
Effect of a program to improve oral communication skills on self-
} reported anxiety and stress

\author{
Antônio Alexandre de Medeiros Lira' (1), Daniel Lucas Picanço Marchand ${ }^{1}$ (D), Lucas Sávio Rodrigues Carvalho² (i), \\ Mauriceia Cassol' 1 (1)
}

\section{RESUMO}

Objetivo: Verificar os efeitos de um programa fonoaudiológico de aprimoramento das habilidades de comunicação oral, por meio da mensuração dos índices autorreferidos de ansiedade e estresse. Métodos: Trata-se de um ensaio clínico randomizado controlado. Os participantes da pesquisa foram divididos aleatoriamente em dois grupos: grupo intervenção (GI) e grupo controle (GC). O GI participou de seis oficinas de aprimoramento das habilidades de comunicação oral. O GC participou de uma única oficina sobre saúde vocal. Foram aplicados, antes da primeira e após a última oficina, os protocolos: Questionário de caracterização da amostra, Self Statements During Public-Speaking Scale (SSPS), Perceived Stress Scale (PSS) e o Cuestinário de Ansiedad Social para Adultos (CASO). Após seis meses, foi realizado um follow-up. Resultados: O GI apresentou, após o aprimoramento, redução significativa dos escores de ansiedade no CASO, de forma geral, e em todos os parâmetros do protocolo. Os valores da PSS referentes ao estresse demonstraram aumento da autoavaliação positiva e diminuição da autoavaliação negativa. Já na autoavaliação ao falar em público, foi verificado, na SSPS, aumento da pontuação geral, em relação ao GC. Conclusão: O programa de aprimoramento fonoaudiológico das habilidades de comunicação oral promoveu a diminuição nos índices de ansiedade e estresse autorreferidos, aumentando a autopercepção positiva ao falar em público. O presente estudo foi registrado no Registro Brasileiro de Ensaios Clínicos (ReBEC), sob o identificador primário RBR-37r3S2.

Palavras-chave: Fonoaudiologia; Comunicação; Ansiedade; Estresse; Treinamento de voz

\begin{abstract}
Purpose: The present study aimed to verify the effects of a speech therapy program to improve oral communication skills by measuring self-reported anxiety and stress indexes. Methods: This is a randomized controlled clinical trial approved by the Research Ethics Committee under number $2,729,273$. Research participants were randomly divided into two groups: intervention group (IG) and control group (CG). The IG participated in six workshops to improve oral communication skills. The CG participated in a single vocal health workshop. The protocols were applied before the first and after the last workshop: Sample characterization questionnaire, Self Statements During Public-Speaking Scale (SSPS), Perceived Stress Scale (PSS), and the Social Anxiety Questionnaire for Adults (SAQA). After six months, a follow-up was carried. Results: The IG showed, after training, a significant reduction of anxiety scores in the SAQA in general, and in all protocol parameters. The PSS values related to stress demonstrated an increase in positive self-assessment and a decrease in negative selfassessment. In the self-assessment when speaking in public by the SSPS, there was an increase in the overall score in relation to the CG. Conclusion: The speech therapy improvement program for oral communication skills promoted a decrease in self-reported anxiety and stress levels, increasing positive self-perception when speaking in public. The present study was registered in the Brazilian Registry of Clinical Trials (ReBEC) under the primary identifier RBR-37r3S2.
\end{abstract}

Keywords: Speech therapy; Communication skills; Anxiety; Stress; Voice training

Trabalho realizado na Universidade Federal de Ciências da Saúde de Porto Alegre - UFCSPA - Porto Alegre (RS), Brasil.

${ }^{1}$ Programa de Pós-graduação em Ciências da Reabilitação, Universidade Federal de Ciências da Saúde de Porto Alegre - UFCSPA - Porto Alegre (RS), Brasil. ${ }^{2}$ Curso de Fonoaudiologia, Universidade Federal de Ciências da Saúde de Porto Alegre - UFCSPA - Porto Alegre (RS), Brasil.

Conflito de interesse: Não.

Contribuição dos autores: AAML foi responsável pelo delineamento do estudo, análise de dados, redação do estudo; DLPM foi responsável pela concepção da pesquisa, delineamento do estudo, coleta e análise de dados, redação e revisão do estudo; LSRC foi responsável pela coleta e análise de dados e revisão do estudo; MC foi responsável pela concepção da pesquisa, delineamento do estudo, análise de dados, orientação, redação e revisão do estudo.

Financiamento: CAPES (Coordenação de Aperfeiçoamento de Pessoal de Nível Superior), processo nº 88882.442869/2019-01. CAPES/FAPERGS (Fundação de Amparo à Pesquisa do Estado do Rio Grande do Sul, processo no 88887.162304/2017-00.

Autor correspondente: Antônio Alexandre de Medeiros Lira. E-mail: alexandrelira-al@hotmail.com

Recebido: Julho 15, 2021; Aceito: Outubro 21, 2021 


\section{INTRODUÇÃO}

A comunicação é uma habilidade essencial para o ser humano. Seu processo ocorre na troca de mensagens entre um emissor e um receptor, por meio de canais verbais, vocais e não verbais. Além do seu papel social, a comunicação exerce uma importante função no contexto profissional e de liderança ${ }^{(1)}$. O que falamos, como falamos e o que queremos falar faz total diferença e pode mudar a nossa forma de ser compreendido pelo outro. Assim, o uso eficaz da comunicação pode reduzir o estresse, promover o bem-estar e, portanto, melhorar a qualidade de vida geral ${ }^{(2)}$.

A sociedade contemporânea vive uma realidade que exige cada vez mais a busca por estratégias que viabilizem o progresso pessoal e profissional e a comunicação exerce relevante papel nesse processo ${ }^{(3,4)}$. Aspectos relacionados ao desenvolvimento pessoal e profissional estão envolvidos com o desempenho comunicacional, tanto de estudantes, como de profissionais, em que $o$ ato de falar publicamente é uma forma de expressar ideias e argumentos ${ }^{(1,5)}$. Para universitários, falar em público é uma exigência que se torna comum em seu processo de formação. $\mathrm{Na}$ apresentação de seminários, trabalhos, ou mesmo durante a aula, $\mathrm{o}$ ato de falar em público pode ser algo desafiador e que pode gerar desconforto ou medo ${ }^{(6,7)}$.

Indivíduos movidos pela ansiedade excessiva ao falar em público podem apresentar tom de voz mais agudo, ressonância laringofaríngea, quebras na frequência, aumento da tensão muscular, incoordenação pneumofonoarticulatória, disfluência, entre outras manifestações ${ }^{(8)}$. Essas alterações podem estar relacionadas à ativação do sistema cerebral de defesa, ocasionando tensão muscular e alterações fisiológicas hormonais ${ }^{(9-1)}$. Em resposta a um fator estressor, o corpo ativa vários processos, com o objetivo de preservar a vida e restaurar a homeostase. Fatores físicos e psicológicos são responsáveis, tanto de forma aguda, quanto crônica, por ativarem reações de estresse no corpo $^{(12)}$.

Falar em público pode ser ainda um fator gerador de estresse ao falante. $\mathrm{O}$ estresse excessivo pode trazer alterações psicológicas com efeitos cognitivos e emocionais, como diminuição da atenção, concentração e memória de curto prazo. Esses efeitos podem interferir diretamente na organização da apresentação do falante, além de diminuir a capacidade de tomar decisões, impactando a clareza de informações e o conteúdo da comunicação. Em relação aos aspectos emocionais, o excesso de estresse gera tensão muscular de forma generalizada e aumenta o risco de tensão laríngea ${ }^{(11-13)}$.

A autopercepção do indivíduo é essencial para que ele possa ajustar a sua comunicação, no contexto pessoal e profissional e, assim, transmitir informações claras e objetivas. Como uma forma de mensurar a autopercepção do sujeito, a utilização de escalas é amplamente aceita. Por serem de fácil aplicação, baixo custo e apresentarem resultados de forma rápida e concisa, os protocolos de autoavaliação são uma alternativa para verificação de padrões comunicacionais. Porém, esses instrumentos podem apresentar limitações, uma vez que os eventos relacionados à própria percepção do sujeito sobre si mesmo podem variar de acordo com o contexto do momento da avaliação e do elemento a ser avaliado ${ }^{(14-16)}$.

Considerando as mudanças causadas pelo crescimento do mercado de trabalho atual, a busca pelo aprimoramento das habilidades de comunicação tornou-se um diferencial para que o indivíduo venha a ter maiores oportunidades após a sua formação ${ }^{(1,2)}$. A Fonoaudiologia é uma ciência que tem a comunicação como objeto de estudo e, desta forma, vem estudando, pesquisando e aprimorando a temática do falar em público, com o objetivo de um entendimento sobre o treinamento e aprimoramento das habilidades comunicativas envolvidas nesse processo ${ }^{(3,4)}$. Achados apontam que o aprimoramento das habilidades de comunicação oral realizado por fonoaudiólogos permite que o indivíduo obtenha autoconfiança na utilização dos aspectos da expressividade comunicativa ${ }^{(3,17)}$.

Considerando a importância da utilização consciente da comunicação em situações de falar em público por universitários, o objetivo do presente estudo foi verificar os efeitos de um programa fonoaudiológico de aprimoramento das habilidades de comunicação oral, por meio da mensuração dos índices autorreferidos de ansiedade e estresse.

\section{MÉTODO}

\section{Participantes}

Esta pesquisa foi aprovada pelo Comitê de Ética em Pesquisa da Universidade Federal de Ciências da Saúde de Porto Alegre - UFCSPA, sob o parecer n ${ }^{\circ}$ 2.729.273 e registrada no Registro Brasileiro de Ensaios Clínicos - ReBEC, sob o identificador primário RBR-37r3s2. Todos os participantes foram voluntários e assinaram o Termo de Consentimento Livre e Esclarecido. Participaram deste estudo estudantes universitários de cursos diversos de universidades públicas e privadas do Sul do Brasil.

Foi realizado cálculo amostral com base no estresse e sua variação, por meio de intervenções alicerçadas no ajuste comunicacional $^{(11)}$, que identificou uma variância explicada de $18,49 \%(\mathrm{r}=0,43)$. Foi estimado um número inicial de 46 participantes, sendo 23 por grupo.

Trata-se de um estudo tipo ensaio clínico randomizado controlado e os estudantes universitários foram convidados para participar da pesquisa por meio de contato pessoal e mídias sociais. Os critérios de inclusão foram: idade entre 18 e 30 anos; ser estudante universitário e assinar o Termo de Consentimento Livre e Esclarecido. Foram considerados critérios de exclusão: ter participado de algum treinamento em oratória ou similar; exercer atividade regular que envolvesse falar em público; indivíduos com diagnóstico médico ou psicológico de transtorno ou fobia social; ter frequentado menos que $75 \%$ das oficinas fonoaudiológicas durante o estudo.

\section{Materiais e procedimentos}

As coletas ocorreram no período de maio a dezembro de 2019, em uma sala de aula da instituição de origem. Inicialmente, foi realizada uma triagem psicológica, por meio da Escala de Ansiedade Social de Liebowitz (LSAS - Liebowitz Social Anxiety Scale $)^{(14)}$, por uma psicóloga com experiência na área. A LSAS é um instrumento de rastreio do transtorno de ansiedade social (TAS), alteração psicológica caracterizada pelo medo acentuado na realização de algumas atividades, em que o indivíduo pode apresentar um comportamento de esquiva fóbica. O objetivo dessa triagem foi verificar a existência de transtorno ou fobia social. Os participantes considerados aptos após a aplicação da 
LSAS responderam aos seguintes questionários: Questionário de caracterização da amostra, SelfStatements During Public-Speaking Scale - SSPS (Escala de Autoavaliação ao Falar em Público), Perceived Stress Scale - PSS (Escala de Estresse Percebido) e o Cuestionario de Ansiedad Social para Adultos - CASO (Questionário de Ansiedade Social para Adultos).

O questionário de caracterização da amostra foi composto por dados pessoais, como nome, idade, sexo, estado civil, curso de graduação, período de formação até o presente momento e uso de medicamentos - visto que este pode influenciar as variáveis pesquisadas.

A $\operatorname{SSPS}^{(9,16)}$ é fundamentada nas teorias cognitivas que tratam a ansiedade social como resultado da autopercepção negativa em relação a si e aos outros. Trata-se de uma escala autoaplicável, traduzida e validada para o português brasileiro, composta por duas subescalas: uma de autoavaliação positiva e outra de autoavaliação negativa. Cada subescala é formada por cinco questões e pontuada de 0 a 5 . Quanto maior for a pontuação geral, menor é o índice de ansiedade ao falar em público. Um estudo considerou como valor de referência o escore acima de 32 pontos ao se referir à autoavaliação positiva ao falar em público e, abaixo deste valor, à autoavaliação negativa ${ }^{(10)}$.

$\mathrm{APSS}^{(13)}$ tem como objetivo mensurar os índices de estresse percebido, provocados pelas alterações fisiológicas decorrentes de um fator estressor. Trata-se de uma escala com escore geral entre 0 e 56 pontos, composta por 14 itens, divididos em sete negativos $(1,2,3,8,11,12$ e 14) e sete positivos $(4,5,6,7,9$, 10 e 13), sendo que o último fator tem sua pontuação invertida para a análise da pontuação geral. Essa escala pode ser utilizada em vários grupos etários, de adolescentes a idosos, e não possui questões específicas quanto ao contexto em que estão inseridos. Quanto maior a pontuação apresentada pelo indivíduo, maior estresse é percebido por ele. A literatura ${ }^{(13)}$ apresenta como ponto de corte dessa escala valores acima de $50 \%$ do seu escore total.

Como forma de avaliar a ansiedade, foi utilizado o Questionário de Ansiedade Social para Adultos (Cuestinário de Ansiedad Social para Adultos - CASO) ${ }^{(18)}$. O questionário foi traduzido e validado no Brasil e seu escore varia entre 0 e 150 pontos, sendo que o sujeito que apresenta mais que 92 pontos, de forma geral seria caracterizado com ansiedade excessiva. As propriedades psicométricas do CASO são eficazes em âmbito global, porém, sua especificidade é direcionada a estudantes universitários. A versão brasileira do CASO é composta por 30 itens, com questões pontuadas em uma escala de Likert, em que 1 está relacionado a nenhum ou muito pouco mal-estar, tensão ou nervosismo e 5 , a muito ou muitíssimo mal-estar, tensão ou nervosismo. O CASO avalia cinco dimensões da ansiedade social, compostas por seis questões cada: falar em público/Interação com pessoas em posição de autoridade; interação com pessoas desconhecidas; interação com o sexo oposto; expressão assertiva de incômodo, desagrado ou raiva; ficar em evidência ou fazer papel de ridículo.

\section{Alocação de grupos}

Os participantes da pesquisa foram pareados e alocados por meio de randomização estratificada, levando-se em consideração a idade, o sexo e a nota geral no SSPS. A randomização foi realizada pela plataforma random.org em dois grupos: grupo intervenção (GI) e grupo controle (GC).

\section{Oficinas}

O GI participou de seis oficinas de aprimoramento das habilidades de comunicação oral, ministradas por um fonoaudiólogo especialista em voz, com mais de cinco anos de experiência na área. As oficinas abordaram aspectos da comunicação verbal, não verbal e vocal, com os seguintes conteúdos: Oficina 1: Autoconhecimento vocal; Oficina 2: Respiração; Oficina 3: Articulação e ritmo de fala; Oficina 4: Coordenação pneumofonoarticulatória; Oficina 5: Expressividade vocal e Oficina 6: Comunicação não verbal. As oficinas eram semanais, tinham a duração de duas horas e foram realizadas em pequenos grupos de até cinco pessoas, para que os participantes não se habituassem a falar para um grande número de pessoas. A escolha dos conteúdos abordados durante as oficinas com o GI foi elaborada com base na literatura, levandose em consideração as principais habilidades e competências comunicativas necessárias à comunicação mais clara e objetiva ${ }^{(19-22)}$.

Em todas as oficinas, os participantes do GI receberam um treinamento teórico sobre o conteúdo proposto, com o objetivo de promover uma comunicação mais consciente e, assim, obter maior adesão e motivação para execução dos exercícios fonoaudiológicos e participação nas exposições orais, por meio de leituras e de fala espontânea. Os sujeitos do GI utilizavam os recursos comunicacionais abordados nas oficinas, realizando atividades que não necessitavam de conhecimento prévio, como jogos de improviso e contação de histórias. O Quadro 1 ilustra a realização das oficinas práticas com o GI.

O GC participou de uma única oficina sobre saúde vocal, com duração de duas horas, em que foram apresentados os cuidados com a voz.

Após as seis oficinas de aprimoramento das habilidades comunicacionais do GI e da oficina sobre saúde vocal com o $\mathrm{GC}$, foram reaplicados os mesmos questionários realizados no período pré-intervenção.

Após seis meses do término da realização das oficinas de aprimoramento das habilidades comunicacionais, foi realizado um follow-up com todos os participantes (GC e GI) para verificar a manutenção dos resultados e a eficácia desse programa nos índices de ansiedade e estresse. Os sujeitos de ambos os grupos foram novamente convidados a realizar uma autoavalição dos parâmetros de ansiedade, estresse e falar em público, por meio dos protocolos CASO, PSS e SSPS.

\section{Análise de dados}

Os dados foram analisados de forma descritiva e inferencial. Utilizou-se o software SPSS 25.0.

A descrição das variáveis qualitativas nominais foi realizada mediante frequência relativa e frequência absoluta. A descrição das variáveis quantitativas e qualitativas ordinais foi realizada por meio de medidas de variabilidade (desvio padrão), tendência central (média e mediana) e posição (mínimo, máximo, primeiro quartil e terceiro quartil).

As variáveis quantitativas passaram por uma análise da homogeneidade da distribuição com o teste Shapiro Wilk e apenas a variável idade não apresentou distribuição normal. Dessa forma, a comparação entre os grupos em função das variáveis quantitativas normais foi realizada com o teste $t$ de Student para amostras pareadas. Considerou-se o nível de significância de 5\% para todas as análises inferenciais. 
Quadro 1. Conteúdos e atividades realizadas nas oficinas de aprimoramento da comunicação no grupo intervenção

\begin{tabular}{|c|c|c|}
\hline & Conteúdo & Estratégias e atividades \\
\hline Oficina 1 & $\begin{array}{c}\text { Autoconhecimento } \\
\text { vocal }\end{array}$ & $\begin{array}{l}\text { Intervenção pedagógica: Conteúdo expositivo sobre comunicação } \\
\text { - Tipos de comunicação } \\
\text { - Elementos da comunicação } \\
\text { - Interação e rapport }\end{array}$ \\
\hline Oficina 2 & Respiração & $\begin{array}{l}\text { Intervenção pedagógica: Conteúdo expositivo sobre respiração e sua importância durante a } \\
\text { fala e no controle da ansiedade } \\
\text { Intervenção Respiratória: } \\
\text { • Avaliação e ajuste do tipo respiratório } \\
\text { - Estímulo à autopercepção e propriocepção corporal durante a respiração } \\
\text { - Emissão de sons fricativos em tempo máximo de fonação } \\
\text { - Emissão de sons fricativos de forma fracionada } \\
\text { - Controle inspiratório e expiratório } \\
\text { - Atenção respiratória em meio a múltiplos estímulos }\end{array}$ \\
\hline Oficina 3 & $\begin{array}{c}\text { Articulação e ritmo } \\
\text { de fala }\end{array}$ & $\begin{array}{l}\text { Intervenção pedagógica: Conteúdo expositivo sobre a importância da articulação para uma } \\
\text { comunicação clara e eficaz } \\
\text { Intervenção Articulatória: } \\
\text { - Realização de exercícios articulatórios (estalar a língua, ler trava-língua, diadococinesia, } \\
\text { sobrearticulação com rolhas e leitura apenas de vogais em textos musicais) } \\
\text { - Leitura individual com foco na articulação e ritmo da fala }\end{array}$ \\
\hline Oficina 4 & $\begin{array}{l}\text { Coordenação } \\
\text { pneumofonoarticulatória } \\
\text { (CPFA) }\end{array}$ & $\begin{array}{l}\text { Intervenção pedagógica: Conteúdo expositivo sobre a execução da coordenação } \\
\text { pneumofonoarticulatória } \\
\text { Intervenção fonoarticulatória: } \\
\text { • Suporte respiratório e função vocal: Inspirar em } 4 \text { s, segurar } 5 \text { s e soltar com emissão de } \\
\text { palavras, frases e textos }\end{array}$ \\
\hline Oficina 5 & $\begin{array}{l}\text { Expressividade vocal: } \\
\text { Uso de pausas, } \\
\text { ênfase e entonação }\end{array}$ & $\begin{array}{l}\text { Intervenção pedagógica: A importância da utilização de pausas, ênfases e entonação durante } \\
\text { o discurso } \\
\text { Intervenção na expressividade: } \\
\text { • Condução de treinamento usando recursos vocais (pausa, ênfase e entonação), leitura em } \\
\text { voz alta a partir de diferentes tipos de texto }\end{array}$ \\
\hline Oficina 6 & $\begin{array}{l}\text { Comunicação não } \\
\text { verbal: } \\
\text { Postura, expressão } \\
\text { facial, gestos, imagem } \\
\text { pessoal e corporal }\end{array}$ & $\begin{array}{l}\text { Intervenção pedagógica: Impacto da comunicação não verbal } \\
\text { • Como demonstrar uma comunicação eficaz por meio da utilização da linguagem corporal } \\
\text { Intervenção na expressão não verbal: } \\
\text { • Apresentação oral de cada participante com duração de } 3 \text { minutos sobre um tema de } \\
\text { conhecimentos gerais com foco no ajuste da postura, equilíbrio na expressão facial e } \\
\text { corporal. }\end{array}$ \\
\hline
\end{tabular}

\section{RESULTADOS}

Participaram do presente estudo 39 estudantes universitários (Figura 1), divididos em dois grupos: grupo controle (GC) - 17 estudantes com média de idade de 21 anos e 9 meses; grupo Intervenção (GI) - 22 estudantes com média de idade de 21 anos e 6 meses. A mediana do GC foi terceiro ano de curso e a do GI esteve entre o segundo e o terceiro ano de curso. Em ambos os grupos foram mais frequentes estudantes solteiros e etnia branca. Verificou-se que os participantes não utilizavam medicamentos que pudessem interferir nas análises.

Verificou-se que houve redução nos escores, do momento pré para o momento pós-intervenção, apenas no GI, para os domínios do Questionário de Ansiedade Social para Adultos, (CASO), sendo significativos o escore total ( $p<0,001)$, interação com sexo oposto $(p=0,001)$, expressão assertiva $(p=0,030)$, fala em público $(\mathrm{p}=0,001)$, interação com desconhecidos $(\mathrm{p}=0,001)$ e fazer papel de ridículo $(\mathrm{p}<0,001)$ (Tabela 1$)$.

Quanto aos escores da Escala de Estresse Percebido (PSS), constatou-se aumento nos valores do momento pré para o momento pós-avaliação, no fator PSS positiva no $\mathrm{GC}(\mathrm{p}=0,031)$ e no GI $(p=0,016)$, e redução nos valores do momento pré para o momento pós-intervenção no fator PSS negativa no GC $(p=0,016)$ e no GI $(p=0,007)$ (Tabela 2$)$.

No que se refere à análise da Escala para Autoavaliação ao Falar em Público (SSPS) após intervenção, observou-se redução nos valores dos fatores SSPS total $(p=0,010)$ e SSPS negativa $(p=0,016)$ no momento pós-intervenção para o $\mathrm{GC}$, e aumento nos valores dos fatores total $(p<0,001)$, positiva $(\mathrm{p}<0,001)$ e negativa $(\mathrm{p}=0,007)$ do momento pré para o momento pós-intervenção no GI (Tabela 3).

As Figuras 2, 3 e 4 apresentam os resultados pré-intervenção, pós-intervenção e follow-up após seis meses da autopercepção da ansiedade ao falar em público (SSPS), da ansiedade (CASO) e do estresse percebido ao falar em público (PSS) nos grupos estudados. Os valores apresentados na Figura 2 demonstram que o aumento na pontuação geral da SSPS no GI, após aprimoramento, foi mantido no follow-up, com pequena diferença na diminuição da média. Já as Figuras 3 e 4, apontam para a diminuição dos escores totais das escalas CASO e PSS no GI, após aprimoramento, com aumento da média geral no follow-up da CASO e diminuição da média total no followup da PSS. 


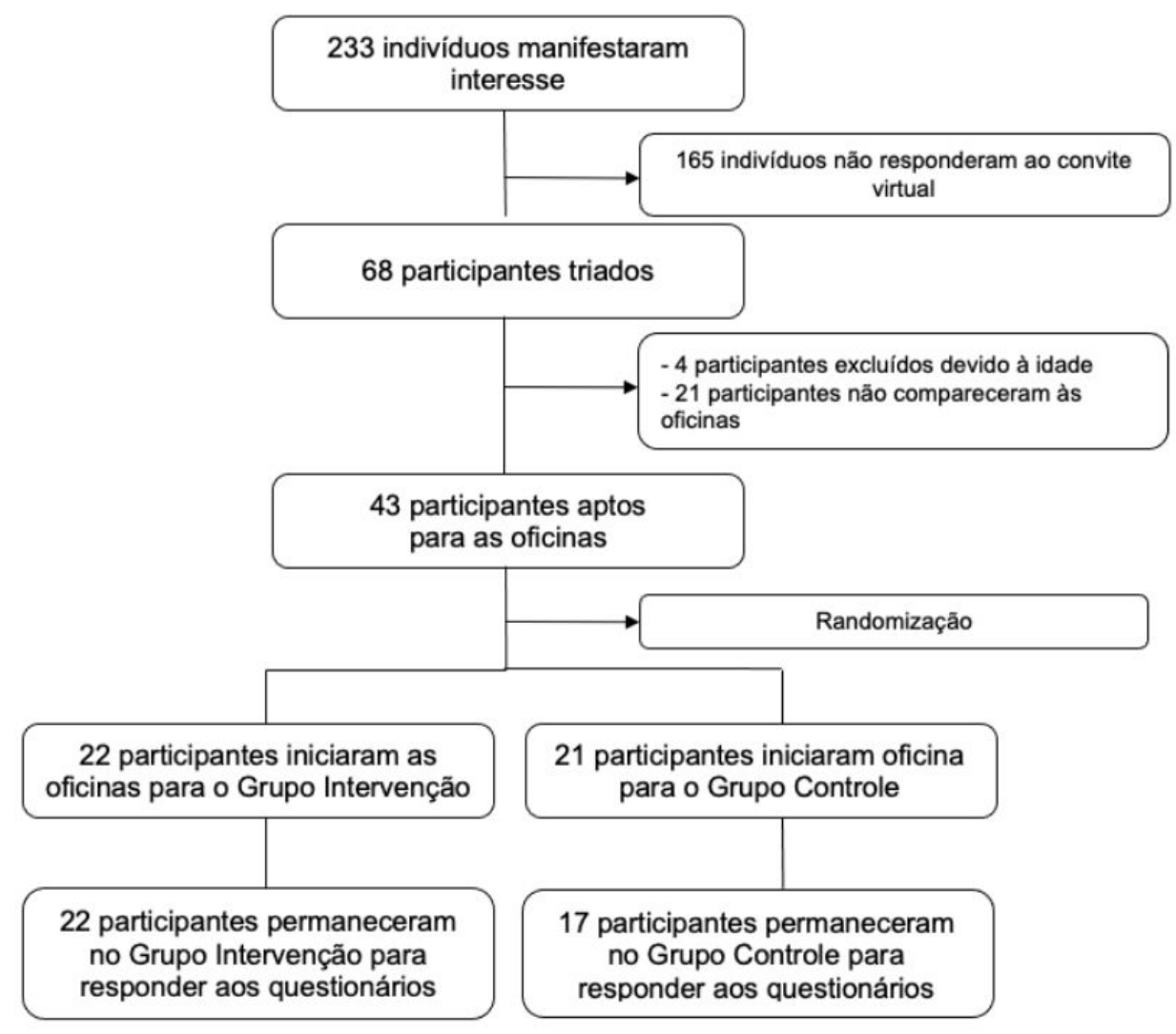

Figura 1. Fluxograma descritivo do processo de amostragem

Tabela 1. Análise do Questionário de Ansiedade Social para Adultos em estudantes universitários, pré e pós-intervenção

\begin{tabular}{|c|c|c|c|c|c|c|}
\hline \multirow{2}{*}{ Variável } & \multirow{2}{*}{ Grupo } & \multicolumn{2}{|c|}{ Pré } & \multicolumn{2}{|c|}{ Pós } & \multirow{2}{*}{ valor de $p$} \\
\hline & & Média & DP & Média & DP & \\
\hline \multirow[t]{2}{*}{ Total } & GC & 102,71 & 20,81 & 95,53 & 27,30 & 0,115 \\
\hline & GI & 101,09 & 19,51 & 83,73 & 24,45 & $<0,001^{*}$ \\
\hline \multirow[t]{2}{*}{ Interação com Sexo Oposto } & GC & 20,24 & 6,56 & 18,76 & 7,72 & 0,091 \\
\hline & Gl & 21,41 & 5,32 & 17,18 & 6,13 & $0,001^{*}$ \\
\hline \multirow[t]{2}{*}{ Expressão Assertiva } & GC & 20,24 & 4,72 & 19,53 & 6,21 & 0,466 \\
\hline & Gl & 20,00 & 4,42 & 18,05 & 5,09 & $0,030^{*}$ \\
\hline \multirow[t]{2}{*}{ Fala em Público } & GC & 22,41 & 4,80 & 21,29 & 5,93 & 0,351 \\
\hline & Gl & 21,41 & 5,73 & 17,55 & 6,71 & $0,001^{*}$ \\
\hline \multirow[t]{2}{*}{ Interação com Desconhecidos } & GC & 18,00 & 4,95 & 16,24 & 5,67 & 0,050 \\
\hline & GI & 17,32 & 5,39 & 13,36 & 4,52 & $0,001^{*}$ \\
\hline \multirow[t]{2}{*}{ Fazer Papel de Ridículo } & GC & 21,82 & 5,05 & 19,71 & 5,64 & 0,135 \\
\hline & $\mathrm{Gl}$ & 20,95 & 4,34 & 17,59 & 5,85 & $<0,001^{*}$ \\
\hline
\end{tabular}

Teste $t$ de Student para amostras pareadas. *Diferença estatisticamente significativa com $\mathrm{p}<0,05$

Legenda: $\mathrm{GC}$ = grupo controle; $\mathrm{Gl}$ = grupo intervenção; $\mathrm{DP}$ = desvio padrão

Tabela 2. Análise da Escala de Estresse Percebido em estudantes universitários, pré e pós-intervenção

\begin{tabular}{|c|c|c|c|c|c|c|}
\hline \multirow{2}{*}{ Variável } & \multirow{2}{*}{ Grupo } & \multicolumn{2}{|c|}{ Pré } & \multicolumn{2}{|c|}{ Pós } & \multirow{2}{*}{ valor de $p$} \\
\hline & & Média & DP & Média & DP & \\
\hline \multirow[t]{2}{*}{ PSS Total } & GC & 31,41 & 3,16 & 28,12 & 9,08 & 0,194 \\
\hline & GI & 33,18 & 3,81 & 30,73 & 8,53 & 0,225 \\
\hline \multirow[t]{2}{*}{ PSS Positiva } & GC & 13,47 & 3,73 & 15,76 & 4,60 & $0,031^{*}$ \\
\hline & GI & 14,50 & 3,35 & 17,00 & 4,93 & $0,016^{*}$ \\
\hline \multirow[t]{2}{*}{ PSS Negativa } & $\mathrm{GC}$ & 17,94 & 4,67 & 12,35 & 5,29 & $0,016^{*}$ \\
\hline & GI & 18,68 & 4,31 & 13,73 & 4,58 & $0,007^{*}$ \\
\hline
\end{tabular}

Teste $t$ de Student para amostras pareadas *Diferença estatisticamente significativa com $p<0,05$

Legenda: PSS = Perceived Stress Scale (Escala de Estresse Percebido); GC = grupo controle; GI = grupo intervenção; DP = desvio padrão 
Tabela 3. Análise da Escala para Autoavaliação ao Falar em Público em estudantes universitários, pré e pós-intervenção

\begin{tabular}{|c|c|c|c|c|c|c|}
\hline \multirow{2}{*}{ Variável } & \multirow{2}{*}{ Grupo } & \multicolumn{2}{|c|}{ Pré } & \multicolumn{2}{|c|}{ Pós } & \multirow{2}{*}{ valor de $p$} \\
\hline & & Média & DP & Média & DP & \\
\hline \multirow[t]{2}{*}{ SSPS Total } & GC & 30,53 & 5,40 & 26,41 & 5,77 & $0,010^{*}$ \\
\hline & GI & 29,55 & 4,92 & 37,36 & 5,74 & $<0,001^{*}$ \\
\hline \multirow[t]{2}{*}{ SSPS Positiva } & GC & 15,06 & 4,96 & 16,82 & 3,67 & 0,194 \\
\hline & GI & 15,77 & 3,48 & 18,59 & 3,70 & $<0,001^{*}$ \\
\hline \multirow[t]{2}{*}{ SSPS Negativa } & GC & 15,47 & 6,40 & 9,59 & 4,02 & $0,016^{*}$ \\
\hline & GI & 13,77 & 5,19 & 19,36 & 3,33 & $<0,001^{*}$ \\
\hline
\end{tabular}

Teste $t$ de Student para amostras pareadas. ${ }^{*}$ Diferença estatisticamente significativa com $\mathrm{p}<0,05$

Legenda: SSPS = Self Statements During Public-Speaking Scale (Escala para Autoavaliação ao Falar em Público); GC = grupo controle; GI = grupo intervenção; $\mathrm{DP}=$ desvio padrão

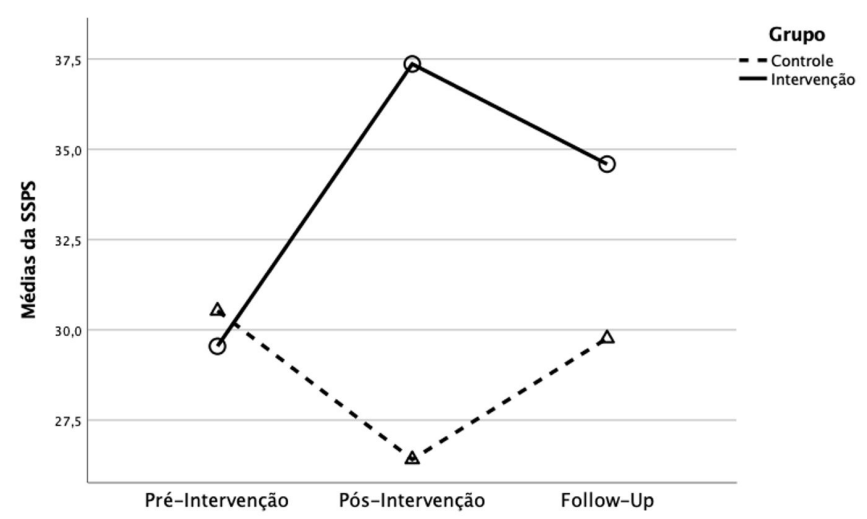

Figura 2. Médias da Escala para Autoavaliação ao Falar em Público em relação aos momentos de aplicação Legenda: SSPS = Self Statements During Public-Speaking Scale (Escala para Autoavaliação ao Falar em Público)

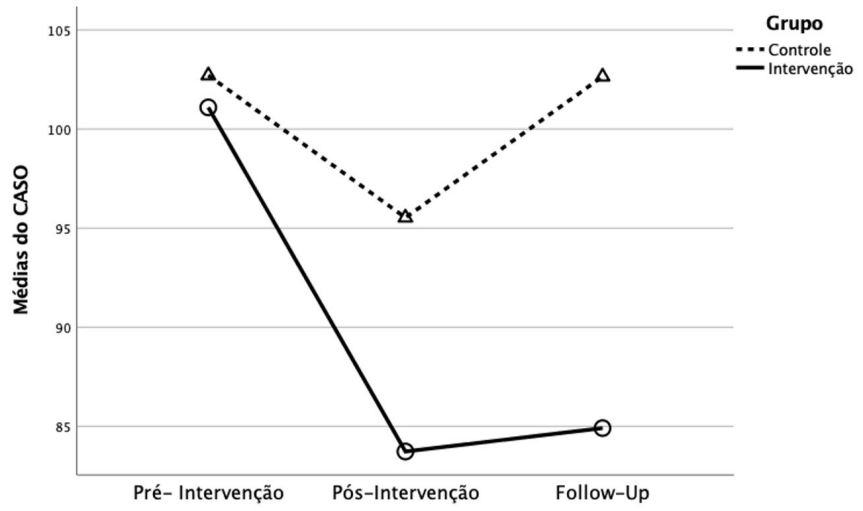

Figura 3. Médias totais do Questionário de Ansiedade Social para Adultos em relação aos momentos de aplicação Legenda: CASO = Cuestionario de Ansiedad Social para Adultos (Questionário de Ansiedade Social para Adultos)

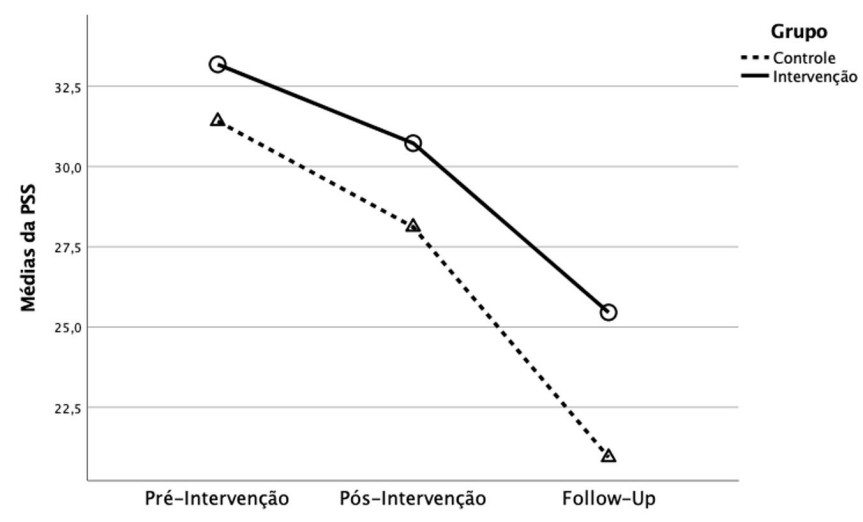

Figura 4. Médias totais da Escala de Estresse Percebido em relação aos momentos de aplicação Legenda: PSS = Perceived Stress Scale (Escala de Estresse Percebido) 


\section{DISCUSSÃO}

O medo de falar em público é o mais prevalente em toda população mundial, segundo estudos epidemiológicos, independentemente de gênero, idade, raça ou classe social, e pode ser considerado algo comum na vida das pessoas. Possivelmente, um ou outro indivíduo já tenha apresentado pelo menos alguma ansiedade relacionada a esse aspecto em um momento de sua vida ${ }^{(5,7,10)}$. Estudo realizado no Brasil com estudantes universitários mostrou que mais de $60 \%$ dos estudantes têm medo de falar em público, fato que pode estar associado à ansiedade excessiva e ter um impacto direto na comunicação $0^{(7,10)}$.

Programas fonoaudiológicos de aprimoramento das habilidades de comunicação oral são realizados em grupo e com objetivos específicos para cada sessão de treinamento. Esses programas abordam aspectos relacionados à orientação e à saúde vocal, treinamento e aprimoramento dos recursos verbais, não verbais e vocais, com a finalidade de promover objetividade, clareza e flexibilidade na comunicação ${ }^{(3)}$.

$\mathrm{O}$ treino dos recursos de ênfase e expressividade comunicativa são realizados por meio da leitura de textos variados e da fala espontânea. Técnicas de respiração, coordenação pneumofonoarticulatória, velocidade de fala, articulação e expressão corporal são utilizados como estratégias para promover mais segurança e credibilidade na comunicação dos falantes. Essas técnicas permitem melhor fluência de leitura, distribuição de pausas e ajuste adequado dos recursos vocais. Além disso, os participantes desses treinamentos referem mais segurança emocional, melhor interação com seus ouvintes e maior consciência corporal durante suas apresentações ${ }^{(4,21)}$.

Pesquisas envolvendo o aprimoramento dessas habilidades e realizadas por fonoaudiólogos têm demonstrado resultados positivos na percepção dos aspectos de fala, corporais, emocionais e interacionais ${ }^{(20-22)}$

Os resultados do presente estudo apontaram que apenas os estudantes universitários do GI tiveram redução significativa dos escores de ansiedade, observados no CASO, após as oficinas fonoaudiológicas de aprimoramento das habilidades de comunicação oral, de forma geral, e no que se refere aos aspectos de interação com o sexo oposto, expressão assertiva, falar em público, interação com desconhecidos e medo de fazer o papel de ridículo.

De acordo com estudos realizados pelos autores do protocolo CASO, foi definido um ponto de corte para determinação do grau de ansiedade em relação à pontuação geral das respostas. Os valores acima de 92 pontos são classificados como de alta ansiedade, em relação aos valores abaixo dessa pontuação ${ }^{(18)}$. Desta forma, além da diminuição significativa dos valores no GI após a realização das oficinas em relação ao momento préintervenção, os estudantes permaneceram com média abaixo do ponto de corte, demonstrando, assim, estarem menos ansiosos naquele determinado momento. Já o GC, além de não apresentar diminuição significativa após a oficina de saúde vocal, ainda mostrou valores superiores ao ponto de corte estabelecido, o que aponta para níveis maiores de ansiedade. É importante ressaltar que antes da realização das oficinas os valores da pontuação geral do CASO apresentavam níveis elevados de ansiedade em ambos os grupos.

Na literatura, os achados referem que, quanto maiores os níveis de ansiedade, mais impacto negativo poderá ser demonstrado durante a performance comunicativa do sujeito ${ }^{(6,8)}$. No entanto, a realização de programas de aprimoramento das habilidades de comunicação oral proporciona ao indivíduo autoconfiança na utilização dos aspectos da expressividade da comunicação e, assim, apresentar diminuição de fatores associados ao medo e ansiedade, demonstrando uma comunicação mais clara e objetiva $^{(19-23)}$.

Pôde-se observar que a diminuição dos escores de ansiedade, obtida por meio do aprimoramento das habilidades comunicacionais, influenciou não apenas o parâmetro "falar em público" da escala CASO, mas todos os demais parâmetros. Portanto, é possível sugerir que quando apresentamos melhor desempenho na habilidade de comunicação, os resultados também se refletem em outros aspectos relacionados com a interação social, seja com o sexo oposto ou com desconhecidos, indicando que o aumento na autoconfiança em se comunicar pode promover maior qualidade de vida ao indivíduo, do ponto de vista social. Com a diminuição dos escores de ansiedade nos parâmetros relacionados à "expressão assertiva" e "fazer o papel de ridículo", espera-se que os estudantes que participaram do programa de aprimoramento das habilidades de comunicação apresentem pensamentos mais positivos e, assim, demonstrem uma expressividade comunicativa assertiva.

A utilização de estratégias em um programa de aprimoramento comunicativo em estudantes universitários promove o uso consciente dessas habilidades e, desse modo, quando confrontados com situações nas quais seja necessário falar em público, são capazes de demonstrar maior segurança, clareza das informações e credibilidade frente aos seus interlocutores.

Pesquisa realizada com universitários de cursos da área da saúde, avaliou a eficácia de um programa de aprimoramento fonoaudiológico das habilidades de comunicação oral, por meio de oficinas com foco na performance comunicativa, durante oito semanas. Todos os resultados evidenciaram evolução positiva e foram estatisticamente significativos do momento pré para o momento pós-intervenção, tanto na avaliação de juízes fonoaudiólogos, quanto nos testes de autoavalição, concordando com os resultados do presente estudo ${ }^{(24)}$.

No que diz respeito ao efeito do estresse na situação de falar em público, a literatura aponta vários efeitos psicológicos, cognitivos e emocionais no falante, que interferem diretamente em sua performance comunicativa ${ }^{(12,13)}$. Neste estudo, os resultados obtidos com o treinamento das habilidades comunicacionais relacionados ao estresse ao falar em público revelaram, por meio da PSS, dados positivos e significativos nos grupos GC e GI nas subescalas positiva e negativa. Pode-se inferir que o treinamento das habilidades comunicativas, no GI, promoveu um efeito positivo na diminuição da percepção do falar em público como um agente estressor e, assim, estimulou maior equilíbrio das emoções em uma situação de exposição ao outro, além da autopercepção positiva sobre bem-estar, levando ao aumento da autoestima. Esses resultados podem refletir diretamente no desempenho comunicativo do GI, com uma apresentação mais organizada, maior concentração no momento de sua apresentação e diminuição da tensão característica do estresse ${ }^{(11,13)}$.

Em relação à autoavaliação em falar em público, os estudantes universitários do GI apresentaram resultados significativos, de forma geral, e nos parâmetros positivos e negativos da SSPS, em relação ao GC. Estudo que aplicou a SSPS como forma de autoavalição da ansiedade ao falar em público utilizou, como ponto de corte para determinar alta ansiedade em se comunicar, valores abaixo de 32 pontos na pontuação geral. Já os valores 
acima dessa pontuação, representariam autoavaliação mais positiva $^{(9,25)}$. Portanto, apesar de o GC, após a oficina de saúde vocal, apresentar valores significativos em relação ao momento pré-intervenção, a média do escore geral ficou em 27 pontos, o que, de acordo com a literatura utilizada, demonstra autoavaliação mais negativa ao falar em público. Todavia, o GI apresentou aumento dos valores de forma significativa, após a realização do aprimoramento fonoaudiológico das habilidades de comunicação oral, obtendo a média de 36,91 pontos no escore geral, o que sugere a autoavaliação mais positiva e menores níveis de percepção de ansiedade ao falar em público ${ }^{(9,16)}$.

$\mathrm{O}$ aumento da pontuação na subescala positiva na SSPS, associada à diminuição dos escores de ansiedade no CASO, pode demonstrar diminuição da percepção de ansiedade ao se comunicar em público, atitudes mais positivas frente a situações que exigem comunicação e autoconfiança perante a interação com o outro. De acordo com os escores apresentados, pela subescala negativa da SSPS, os escores dos indivíduos do GC foram reduzidos significativamente, enquanto, no GI, esses valores elevaram. Esses dados demonstram que o treinamento das habilidades comunicativas, além de promover o domínio e uso consciente dos recursos comunicativos, foi relevante para a autopercepção do padrão de comunicação e autoanálise. A autoavaliação era, inicialmente, negativa, mas, ao longo do processo de aprimoramento fonoaudiológico, com a promoção de um novo padrão de comunicação, tornou-se mais positiva.

Dentre os aspectos positivos e negativos, a autoavalição positiva da comunicação está associada ao melhor desempenho profissional $^{(26)}$. A ansiedade relacionada à comunicação, em estudantes universitários, conduz a uma atitude passiva em seus estudos e à falta de interesse em aprender, o que pode afetar seu desempenho acadêmico, resultando em baixo aproveitamento em suas avaliações e gerando uma percepção negativa no outro ${ }^{(27)}$.

Quando estudantes obtêm uma preparação comunicativa, antes ou durante o seu processo de formação, é provável que apresentem resultados mais significativos na sua trajetória acadêmica, visto que uma autoavalição mais positiva da comunicação está relacionada a melhores resultados, de acordo com a literatura ${ }^{(26,27)}$.

Algumas pessoas apresentam dificuldade para falar em público, por fobia e questões psicológicas, mas, grande parte dos indivíduos que são maus oradores demonstram dificuldades apenas nas suas habilidades de comunicação, como respiração, voz, dicção, postura e gestos. Esses sujeitos evitam falar em público por possuírem autopercepção negativa de suas habilidades comunicativas e por nunca terem realizado qualquer tipo de treinamento de comunicação $\mathrm{O}^{(3,11)}$.

Intervenções no aprimoramento das habilidades de comunicação, como a realizada neste estudo, reduzem as dificuldades de comunicação das pessoas, promovem uma imagem mais positiva e, assim, ajudam a eliminar as barreiras que as afastam de uma interação eficaz no seu ambiente acadêmico e profissional. Em razão de sua visão ampla da comunicação humana, o fonoaudiólogo é o profissional mais habilitado para fornecer treinamento às pessoas que apresentam dificuldades para falar em público ${ }^{(3,4,20,24)}$.

No que diz respeito aos resultados do follow-up, após seis meses do período de intervenção, os participantes do GI demonstraram melhores pontuações, comparados aos do ao GC, nos parâmetros relacionados à ansiedade em falar em público (CASO e SSPS). Isso pode ter ocorrido devido a um aumento da autopercepção das habilidades de comunicação oral que foram aprimoradas durante as oficinas.

No GC, os valores obtidos foram semelhantes aos dos dados coletados no momento pré-intervenção, em ambos os protocolos, demonstrando que os sujeitos ainda apresentavam níveis de ansiedade para falar em público. Nos parâmetros relacionados à autopercepção do estresse, não houve diferença estatística entre o grupo intervenção e o grupo controle, nos três momentos, o que pode ser justificado pelo fato de os estudantes estarem em constante período de avaliação acadêmica e o momento de aplicação dos protocolos pode ter interferido nas respostas em relação ao estresse.

É importante ressaltar que a realização da autoavaliação da comunicação promove a autorregulação, fornece a sensação de continuidade, influencia a percepção social, determina o comportamento em relações sociais e contribui para uma projeção de autoimagem consistente e relevante para o outro. A autoavaliação de estudantes universitários está associada a fatores positivos em sua trajetória acadêmica ${ }^{(15,26,28)}$

Os resultados do presente estudo foram avaliados de forma geral após todas as oficinas de aprimoramento fonoaudiológico. Todos os parâmetros comunicacionais foram positivos para o alcance desses resultados, mas pode-se destacar o parâmetro da respiração com maior relação positiva dentre todos os aspectos abordados nas oficinas. A literatura aponta que a utilização da respiração de forma consciente e equilibrada, durante a comunicação oral, é fundamental para que a mensagem transmitida seja clara e que o falante demonstre maior controle dos níveis de ansiedade ao se comunicar ${ }^{(22,24)}$. A realização dos exercícios respiratórios e os ajustes dos padrões de respiração durante a fala foram fundamentais para obtenção de maior autoconfiança durante a comunicação oral ${ }^{(20,29)}$.

Com este estudo, pode-se inferir que o aprimoramento das habilidades de comunicação nos estudantes universitários do GI foi eficaz no que diz respeito aos aspectos relacionados à ansiedade ao falar em público. Os resultados apontaram, ainda, que, quando alguém faz uso de uma comunicação mais consciente e assertiva, consegue demonstrar maior domínio sobre os aspectos relacionados à expressividade, promovendo, assim, maior autoconfiança ao se comunicar ${ }^{(23,28)}$. Contudo, não houve verificação dos efeitos individuais de cada oficina. Sendo assim, sugere-se a condução de novos estudos, a fim de avaliar a efetividade de cada oficina sobre medo de falar em público.

\section{CONCLUSÃO}

O programa de aprimoramento fonoaudiológico das habilidades de comunicação oral para os estudantes universitários promoveu a diminuição nos índices de ansiedade e estresse autorreferidos e aumentou a autopercepção positiva ao falar em público.

Os achados contribuem para o conhecimento das limitações provocadas pela ansiedade e estresse na comunicação em público e incentivam a busca por aprimoramentos fonoaudiológicos das habilidades de comunicação oral por estudantes, desde a sua formação até a prática profissional, promovendo uma comunicação assertiva, clara e objetiva. 


\section{AGRADECIMENTOS}

Agradecemos ao Programa de Pós-graduação em Ciências da Reabilitação da Universidade Federal de Ciências da Saúde de Porto Alegre-UFCSPA, pela oportunidade de execução deste trabalho.

\section{REFERÊNCIAS}

1. Banwart M. Communication studies: effective communication leads to effective leadership. New Dir Stud Leadersh. 2020;2020(165):87-97. http://dx.doi.org/10.1002/yd.20371. PMid:32187871.

2. Vertino KA. Effective interpersonal communication: a practical guide to improve your life. Online J Issues Nurs. 2014;19(3):1. http://dx.doi. org/10.3912/OJIN.Vol19No03Man01. PMid:26824149.

3. Breakey LK. Fear of public speaking-the role of the SLP. Semin Speech Lang. 2005;26(2):107-17.

4. Borrego MCM, Behlau M. A mapping of the Speech Language Pathology practice pathway in verbal expressivity in the work of communicative competence. CoDAS. 2018;30(6):e20180054. PMid:30517272.

5. Marinho ACF, Medeiros AM, Pantuza JJ, Teixeira LC. Self-perception of shyness and its relation to aspects of public speaking. CoDAS. 2020;32(5):e20190097. PMid:33053085.

6. Kamridah AHY, Arafah B, Imran N. Correlation between level of anxiety and public speaking performance through systematic learning approach in foreign language. Int J Sci Res. 2016;9(5):1658-63.

7. Pantuza JJ, Alexandre IO, Medeiros AM, Marinho ACF, Teixeira LC. Sense of Coherence and the fear of public speaking in university students. CoDAS. 2020;32(5):e20190071. http://dx.doi.org/10.1590/23171782/20202019071. PMid:33053083.

8. Almeida AAF, Behlau M, Leite JR. Correlation between anxiety and communicative performance. Rev Soc Bras Fonoaudiologia. 2011;16(4):384-9.

9. Hofmann SG, DiBartolo PM. An instrument to assess self-statements during public speaking: scale development and preliminary psycometric properties. Behav Ther. 2000;31(3):499-515. http://dx.doi.org/10.1016/ S0005-7894(00)80027-1. PMid:16763666.

10. Marinho ACF, Medeiros AM, Gama ACC, Teixeira LC. Fear of public speaking: perception of college students and correlates. J Voice. 2017;31(1):127.e7-11. http://dx.doi.org/10.1016/j.jvoice.2015.12.012. PMid:26898522.

11. Carney DR, Cuddy AJC, Yap AJ. Power posing brief nonverbal displays affect neuroendocrine levels and risk tolerance. Psychol Sci. 2010;21(10):1363-8. http://dx.doi.org/10.1177/0956797610383437. PMid:20855902.

12. Russell G, Lightman S. The human stress response. Nat Rev Endocrinol. 2019;15(9):525-34. http://dx.doi.org/10.1038/s41574-019-0228-0. PMid:31249398.

13. Cohen S, Kamarck T, Mermelstein R. A global measure of perceived stress. J Health Soc Behav. 1983;24(4):385-96. http://dx.doi. org/10.2307/2136404. PMid:6668417.

14. Santos LF, Loureiro SR, Souza CJA, Lima OF. Psychometric validation study of the Liebowitz Social Anxiety Scale-Self-reported version for brazilian portuguese. PLoS One. 2013;7(8):1-7.
15. Lopes LW, Vilela EG. Self-assessment and readiness for change in dysphonic patients. CoDAS. 2016;28(3):295-301. http://dx.doi. org/10.1590/2317-1782/20162015111. PMid:27383225.

16. Osório FL, Crippa JAS, Loureiro SR. Cognitive aspects of public speaking: validation of a self-assessment scale for Brazilian university students. Rev Psiquiatr Clin. 2012;2(39):48-54.

17. Bozkurt UB, Erim A, Demiray PÇ. The effects of individual voice training on pre-service Turkish language teachers' speaking performance. Educ Sci Theor Pract. 2018;18(1):151-71.

18. Caballo VE, Salazar IC, Irurtia MJ, Arias B, Hofmann SG. The multidimensional nature and multicultural validity of a new measure of social anxiety: The Social Anxiety Questionnaire for Adults. Behav Ther. 2012;43(2):313-28. http://dx.doi.org/10.1016/j.beth.2011.07.001. PMid:22440068.

19. Goberman AM, Hughes S, Haydock T. Acoustic characteristics of public speaking: anxiety and practice effects. Speech Commun. 2011;53(6):867-76. http://dx.doi.org/10.1016/j.specom.2011.02.005.

20. Trajano FMP, Almeida LNA, Alencar SAL, Braga JEF, Almeida AA. Group voice therapy reduces anxiety in patients with dysphonia. J Voice. 2020;34(5):702-8. http://dx.doi.org/10.1016/j.jvoice.2019.03.003. PMid:30979532.

21. Law T, Lee KY, Ho FN, Vlantis AC, van Hasselt AC, Tong MC. The effectiveness of group voice therapy: a group climate perspective. J Voice. 2012;26(2):e41-8. http://dx.doi.org/10.1016/j.jvoice.2010.12.003. PMid:21550777.

22. Mancuso C, Miltenberger RG. Using habit reversal to decrease filled pauses in public speaking. J Appl Behav Anal. 2016;49(1):188-92. http://dx.doi.org/10.1002/jaba.267. PMid:26947580.

23. Rust C, Gentry WM, Ford H. Assessment of the effect of communication skills training on communication apprehension in first year pharmacy students-a two-year study. Curr Pharm Teach Learn. 2020;12(2):142-6. http://dx.doi.org/10.1016/j.cptl.2019.11.007. PMid:32147155.

24. Celeste LC, Lima AM, Seixas JMA, Silva MA, Silva EM. Communicative performance training in university health students. Audiol Commun Res. 2018;23:e1879.

25. Marinho ACF, Medeiros AM, Lima EP, Pantuza JJ, Teixeira LC. Prevalence and factors associated with fear of public speaking. CoDAS. 2019;31(6):e20180266. http://dx.doi.org/10.1590/23171782/20192018266. PMid:31644711

26. Lira AAM, Borrego MC, Behlau M. Self-assessment of communication resources used by sales representatives and its relation with sales performance. CoDAS. 2019;31(6):e20190067. http://dx.doi. org/10.1590/2317-1782/20192019067. PMid:31721891.

27. Vitasari P, Wahab M, Othman A, Herawan T, Sinnadurai S. The relationship between study anxiety and academic performance among engineering students. Procedia Soc Behav Sci. 2010;8:490-7. http:// dx.doi.org/10.1016/j.sbspro.2010.12.067.

28. Gomes VEFI, Batista DC, Lopes LW, Aquino R, Almeida AA. Symptoms and vocal risk factors in individuals with high and low anxiety. Folia Phoniatr Logop. 2019;71(1):7-15. http://dx.doi.org/10.1159/000494211. PMid:30481774.

29. Kamath A, Urval RP, Shenoy AK. Effect of alternate nostril breathing exercise on experimentally induced anxiety in healthy volunteers using the simulated public speaking model: a randomized controlled pilot study. BioMed Res Int. 2017;2017:2450670. http://dx.doi. org/10.1155/2017/2450670. PMid:29159176. 